Fetal Diagnosis and Therapy

\title{
Cerebral Injury and Neurodevelopmental Impairment after Amnioreduction versus Laser Surgery in Twin-Twin Transfusion Syndrome: A Systematic Review and Meta-Analysis
}

\author{
Jeanine M.M. van Klink ${ }^{a}$ Hendrik M. Koopman ${ }^{b}$ Erik W. van Zwet ${ }^{c}$ \\ Dick Oepkes $^{d}$ Frans J. Walther ${ }^{a}$ Enrico Lopriore ${ }^{a}$ \\ ${ }^{a}$ Division of Neonatology, Department of Pediatrics, ${ }^{b}$ Department of Medical Psychology, ${ }^{\mathrm{c} D e p a r t m e n t}$ of Medical \\ Statistics, and dDivision of Fetal Medicine, Department of Obstetrics, Leiden University Medical Center, \\ Leiden, The Netherlands
}

\section{Key Words}

Amnioreduction - Cerebral injury - Fetoscopic laser surgery • Monochorionic twins $\cdot$ Neurodevelopment $\cdot$ Twin-twin transfusion syndrome

\begin{abstract}
Objective: To estimate the odds of severe cerebral injury and long-term neurodevelopmental impairment in monochorionic twins treated with amnioreduction versus laser surgery for twin-twin transfusion syndrome. Methods: A systematic review and meta-analysis of studies on cerebral injury and long-term impairment after amnioreduction versus laser surgery were conducted. Odds ratios (OR) with their 95\% confidence interval $(\mathrm{Cl})$ were computed. Results: Electronic and manual search identified 63 references. Five studies were included for analysis. We found an ample seven-fold higher risk of severe cerebral injury in live-born children treated with amnioreduction compared to laser (OR 7.69, $95 \% \mathrm{Cl} 2.78-20.0, \mathrm{p}=0.00$ ). In children surviving the neonatal period, the odds were three-times higher following amnioreduction (OR 3.23, 95\% Cl 1.45-7.14, $\mathrm{p}=0.00$ ). Although not significant, monochorionic twins treated with amnioreduction had higher odds of periventricular leukomalacia and intraventricular hemorrhage (OR 2.08, 95\% Cl 0.86-5.00, p =
\end{abstract}

0.10 and $\mathrm{OR} 3.56,95 \% \mathrm{Cl} 0.82-14.29, \mathrm{p}=0.09)$. Unfortunately, there were insufficient long-term outcome data available to estimate the odds of neurodevelopmental impairment. Conclusion: Amnioreduction is associated with an increased risk of severe cerebral injury compared to laser surgery in twintwin transfusion syndrome. Our study highlights a lack of studies focusing on long-term neurodevelopmental outcome. Follow-up into childhood is indispensable to determine outcome in terms of motor, cognitive and socioemotional development.

Copyright $\odot 2012$ S. Karger AG, Basel

\section{Introduction}

Twin-twin transfusion syndrome (TTTS) is a severe complication of monochorionic (MC) twin pregnancies resulting from shunting of blood from one twin (the donor) to the other twin (the recipient) through placental vascular anastomoses. The donor twin becomes hypovolemic and anuric with oligohydramnios. The recipient twin becomes hypervolemic and polyuric with polyhydramnios. TTTS severity can be staged I to V according to Quintero's classification system [1]. Serial amnioreduction of excessive amniotic fluid (AR) and fetoscopic

\section{KARGER}

Fax +41613061234

E-Mail karger@karger.ch

www.karger.com
(C) 2012 S. Karger AG, Basel

$1015-3837 / 13 / 0332-0081 \$ 38.00 / 0$

Accessible online at:

www.karger.com/fdt
Jeanine M.M. van Klink

Department of Pediatrics, Division of Neonatology

Leiden University Medical Center, PO Box 9600

NL-2300 RC Leiden (The Netherlands)

Tel. +31 71526 3925, E-Mail j.m.m.van_klink@lumc.nl 
laser coagulation of the placental vascular anastomoses (FSL) are the two main treatment options in TTTS. There is extensive evidence that serial AR is associated with increased perinatal mortality when compared to laser surgery [2]. Reliable information on long-term impairment in survivors after both interventions is lacking [3].

The objective of the current systematic review and analysis was to estimate the odds of severe cerebral injury and long-term neurodevelopmental impairment in MC twins treated with serial AR compared to laser surgery for TTTS.

\section{Methods}

This systematic review was performed using PRISMA statement: preferred reporting items for systematic reviews and metaanalyses [4]. Inclusion criteria were formulated according to our pre-defined Patient-Intervention-Comparison-Outcome (PICO) question. The patients are live-born MC diamniotic twins with TTTS diagnosed using standard prenatal ultrasound criteria [5]. The intervention refers to serial AR and the comparison is fetoscopic laser coagulation of placental vascular anastomoses. The primary outcome entails severe cerebral injury and long-term neurodevelopmental impairment (NDI) with a follow-up period from pregnancy outcome to childhood:

(1) Severe cerebral injury was defined as intraventricular hemorrhage (IVH) $\geq$ grade III [6], cystic periventricular leukomalacia (cPVL) $\geq$ grade II [7], ventricular dilatation $\geq 97$ th percentile [8], porencephalic cysts, arterial or venous infarction detected on cerebral imaging, that is, cranial ultrasound, computed tomography scan or magnetic resonance imaging.

(2) NDI was defined as cerebral palsy, bilateral blindness, bilateral deafness or cognitive developmental delay $>2$ standard deviations (SD) below the population mean, diagnosed using standardized tests.

Due to an anticipated lack of randomized controlled trials (RCT), we included both randomized and non-randomized studies. Studies that did not match our PICO question were excluded. English language restrictions were applied.

\section{Data Sources}

An electronic literature search was performed with PubMed, MEDLINE and ISI Web of Science (WoS) up to March 2012. Table 1 presents the search strategies for PubMed that were subsequently adapted for use in MEDLINE and ISI WoS. To identify articles not captured by the electronic searches, we hand-searched reference lists of relevant studies.

\section{Study Selection}

Eligibility and methodological quality of the studies were assessed independently by the corresponding (J.M.M.v.K.) and last author (E.L.). The following data were extracted and tabulated: first author, year of publication, study design, country of origin, selection and allocation of patients, data collection, comparability of patients and controls, potential confounders, operational- ization of primary outcome and outcome measurement, efforts to minimize bias, the incidence of severe cerebral injury and NDI in patients and controls $(2 \times 2$ tables $)$ and the length and completeness of follow-up. In case of overlap or duplications in patients between studies, the study with the best overall study quality was included for review. A randomized controlled trial was, a priori, considered the best study design. Disagreements regarding eligibility, methodological quality and data extraction were resolved by discussion and consensus between authors.

\section{Statistical Analysis}

To summarize the results of the selected studies, an Excel spreadsheet was used. We performed statistical analysis using Stata (StataCorp LP, College Station, Tex., USA). Categorical or dichotomous data were meta-analyzed with odds ratios (ORs) and their $95 \%$ confidence intervals (CI), using $2 \times 2$ tables. We used the recommended method to add 0.5 where $2 \times 2$ tables contained cells with zero events, allowing continuity correction. Studies were a priori analyzed into two groups, that is, studies including and studies excluding neonatal deaths in their outcome analysis. Heterogeneity between studies was examined with the inconsistency square $\left(\mathrm{I}^{2}\right)$ statistics, with between-study heterogeneity at $\mathrm{I}^{2} \geq 50 \%$ and $\mathrm{p} \geq 0.05$ [9]. In case of heterogeneity, a random effects model was used [10]. Otherwise, or in case of limited studies to reliably estimate between study variability, a fixed effect model was used. We performed meta-analyses and constructed forest plots to examine the effect of AR compared to laser surgery on severe cerebral injury with separate analyses for CPVL $\geq$ grade II and IVH $\geq$ grade III, and NDI with separate analyses for cerebral palsy, bilateral blindness, deafness and cognitive developmental delay. Publication bias was examined with the construction of a funnel plot and tested for asymmetry with the Egger test [11].

\section{Results}

\section{Study Identification}

Combination of the 4 search strategies revealed 25 references in PubMed, 18 references in MEDLINE and 43 references in ISI WoS. A manual search revealed one additional study for consideration [12]. In total, after removal of duplicates, 63 references were screened. Figure 1 provides a flow diagram with the number of studies screened, assessed for eligibility and included for review according to our PICO question.

We found two other systematic reviews on AR versus laser surgery $[2,13]$. Roberts and colleagues (2008) analyzed one randomized controlled trial to compare AR with laser surgery on short-term perinatal outcome in their Cochrane meta-analysis [2]. To obtain the full range of research to date, we accepted case series as well. Rossi and D'Addario reported on mortality and cerebral anomalies representing the sum of a wide variety of cerebral injuries of varying degrees of severity, regardless of sub- 
Table 1. PubMed search strategies

\begin{tabular}{|c|c|}
\hline Strategy & Mesh and entry terms \\
\hline No. 1 & $\begin{array}{l}\text { “Fetofetal Transfusion”[Mesh] OR "Fetofetal Transfusion”[All Fields] OR “Twin Transfusion Syndrome”[All Fields] OR } \\
\text { "Twin Transfusion Syndromes"[All Fields] OR “Twin Transfusion”[All Fields] OR “Twin Transfusions”[All Fields]) }\end{array}$ \\
\hline No. 2 & $\begin{array}{l}\text { (“Amniocentesis”[Mesh] OR “Amniocentesis”[All Fields] OR “Amniocenteses”[All Fields] OR “Amnioreduction” } \\
\text { [All Fields] OR “Amniodrainage”[All Fields]) }\end{array}$ \\
\hline No. 3 & $\begin{array}{l}\text { "Fetoscopy”[Mesh] OR "Fetoscopy”[All Fields] OR "Fetoscopic Surgeries”[All Fields] OR "Fetoscopic Surgical } \\
\text { Procedures"[All Fields] OR "Intrauterine Laser Treatment”[All Fields] OR "Fetoscopic Laser Surgery”[All Fields] } \\
\text { OR "In Utero Laser Ablation Therapy"[All Fields] OR "Laser Photocoagulation”[All Fields] OR "Laser Surgery" } \\
\text { [All Fields] OR "Endoscopic Laser Surgery"[All Fields] OR "Laser Therapy”[All Fields]) }\end{array}$ \\
\hline No. 4 & $\begin{array}{l}\text { (“Infant”[Mesh] OR “Infant”[All Fields] OR “Infant Development”[All Fields] OR “Child”[All Fields] OR “Child } \\
\text { development"[All Fields] OR "Neurologic Injury"[All Fields] OR "Cerebral Damage”[All Fields] OR } \\
\text { "Neurodevelopmental Outcome"[All Fields] OR "Neurodevelopment"[All Fields] OR "Developmental Follow-Up" } \\
\text { [All Fields]) }\end{array}$ \\
\hline
\end{tabular}

No. 5

No. 1 and No. 2 and No. 3 and No. 4: results: 25

sequent perinatal deaths or overlap in patients between studies, hence susceptible for bias [13-15].

We selected five studies, directly comparing AR with laser surgery on severe cerebral injury and NDI; three comparative studies plus two follow-up studies from the Eurofetus Trial [15, 16]. Although well-designed and highly valuable, the two comparative studies published by Lenclen and colleagues were excluded from analysis, due to an overlap in patients with the two Eurofetus RCT follow-up studies $[14,17]$.

\section{Risk of Bias and Quality Assessment using PRISMA Statement}

Since no randomization took place in the three comparative studies, performance- or selection bias could not be ruled out [18-20]. However, treatment allocation was unlikely correlated with TTTS severity in these studies since allocation to either AR or laser surgery was based on geographical location and treatment availability; consecutive patients were grouped according to treatment center and year of introduction of laser surgery in a center [18]. The RCT performed prospective power analysis and used computer-generated central randomization sequences to maintain adequate allocation concealment $[15,16]$. Four studies employed a prospective design, of which one staged their AR group retrospectively $[15,16$, 19, 20]. All but one were multicenter studies [20]. All studies described techniques for both interventions in detail. Completeness of follow-up ranged from 92 to $100 \%$. Outcome assessment, including timing and fre-

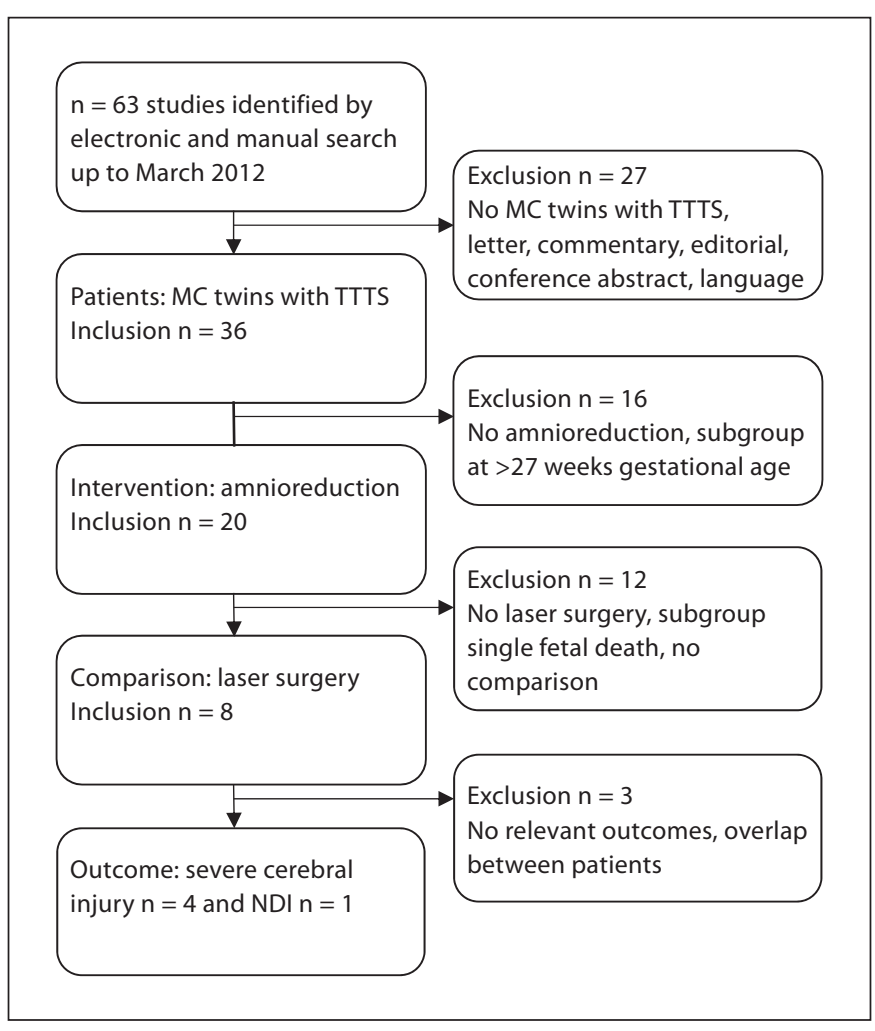

Fig. 1. Flow diagram with the number of studies screened, assessed for eligibility and included for review with exclusion criteria according to our Patient-Intervention-Comparison-Outcome question. 


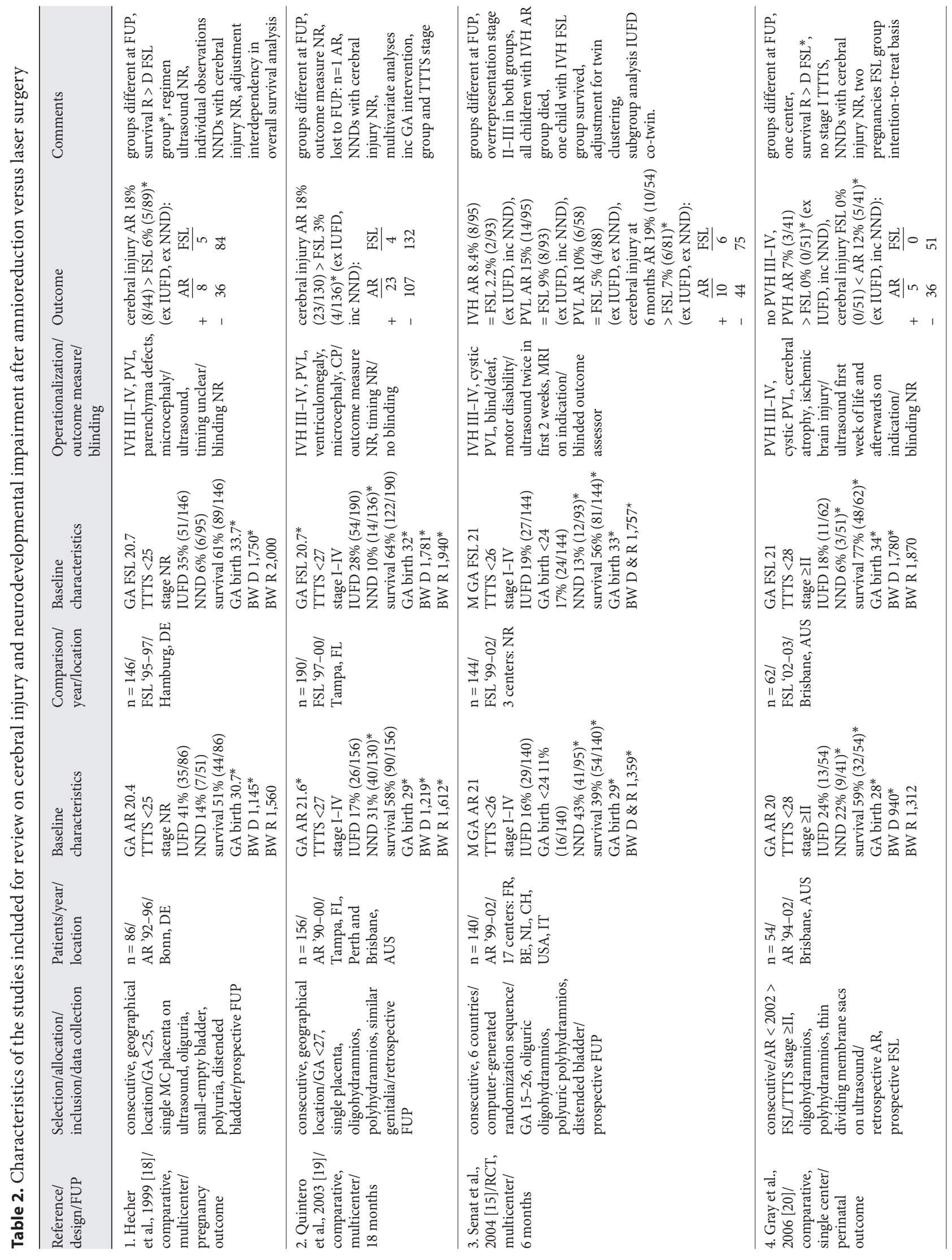




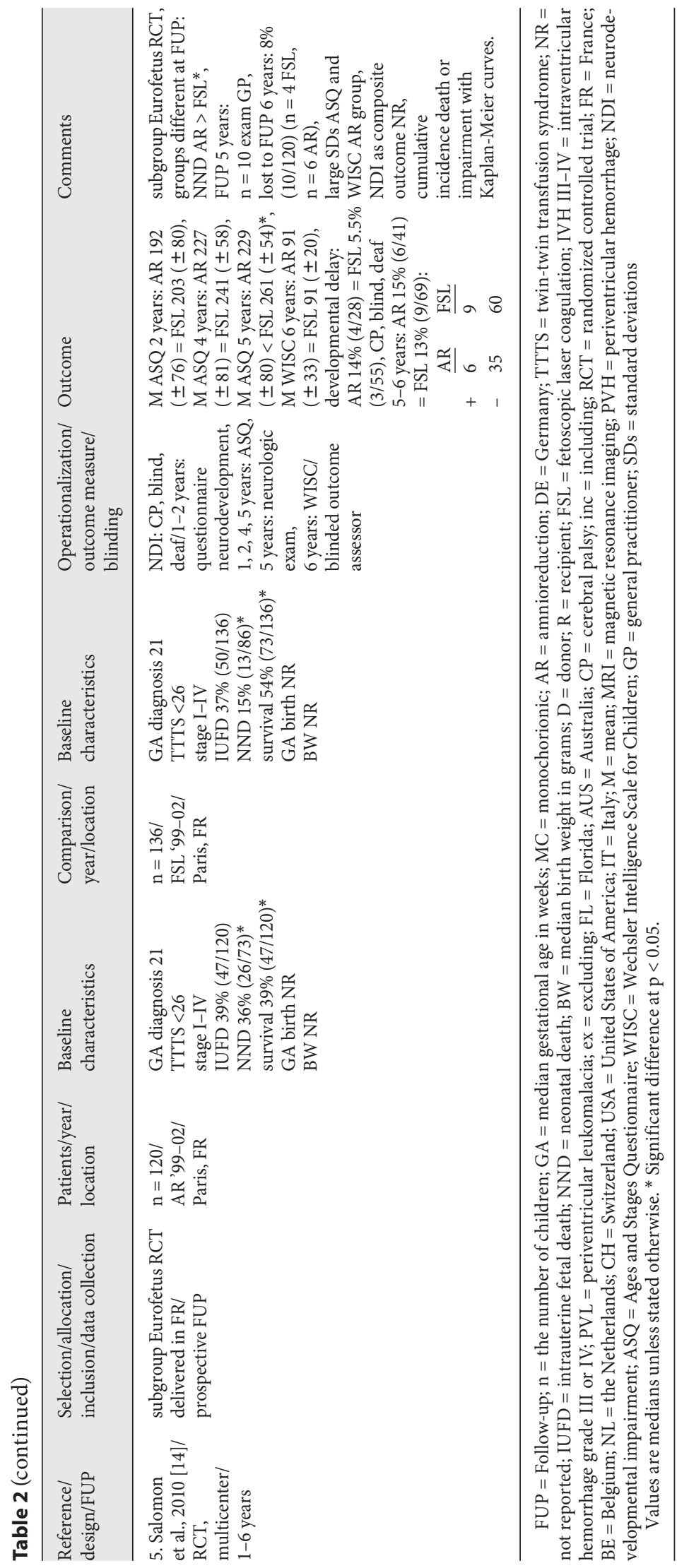

quency of postnatal brain imaging, was fully described in two of five studies and blinded in two $[15,16]$. Four studies accounted for TTTS stage in their comparison of AR to laser, of which one stratified stage on outcome [19]. Two studies reported worse outcomes with increased TTTS stage in both groups; one study found increased TTTS stage associated with poorer outcome in the AR group only [19].

\section{Results of Individual Studies}

Summary data for each intervention group are displayed in table 2. Median gestational age at intervention was 20-22 weeks for both intervention groups and comparable in all but one study; 21.6 weeks at first AR versus 20.7 weeks at laser surgery [19]. This study reported an increased incidence of severe cerebral injury following AR which was related to increased TTTS stage. This effect was not observed in their laser group. All but one study staged TTTS, according to Quintero's classification system $[1,18]$. TTTS stage at intervention was comparable between groups, with limited stage I cases in all studies. Gray and colleagues excluded stage I cases [20]. AR resulted in lower overall survival rates when compared to laser, ranging from 39 to $59 \%$ compared to $54-77 \%$, respectively. Treatment with AR resulted in higher neonatal mortality rates, ranging from 14 to $43 \%$ compared to $6-15 \%$ when treated with laser. Median gestational age at birth was lower with $\mathrm{AR}$, ranging from 28 to 31 versus 32-34 weeks with laser. Accordingly, birth weight of donor and recipient twins was lower in the AR group ranging from 940 to 1,612 versus $1,750-2,000 \mathrm{~g}$ with laser.

\section{Synthesis of Results}

A fixed effect model was used throughout because of the small number of included studies to reliably assess between study variability. To assess the odds of severe cerebral injury in children treated with either AR or laser, data were derived from four studies with 269 children in the AR group versus 357 children in the laser group [15, 18-20]. The odds of severe cerebral injury in live-born children treated with AR were seven- to eight-times higher when compared to children treated with laser (OR 7.69, $95 \%$ CI 2.78-20.0, $\mathrm{p}=0.00$; fig. 2). With subsequent neonatal deaths excluded from outcome analysis, the odds were three-times higher in the AR group compared to the laser group (OR 3.23, 95\% CI 1.45-7.14, $\mathrm{p}=0.00$; fig. 2).

To assess the odds of cPVL $\geq \mathrm{II}$ in live-born children, data were derived from two studies, one RCT and one comparative study, with 136 children in the AR and 144 children in the laser group $[15,20]$. The OR demonstrated 


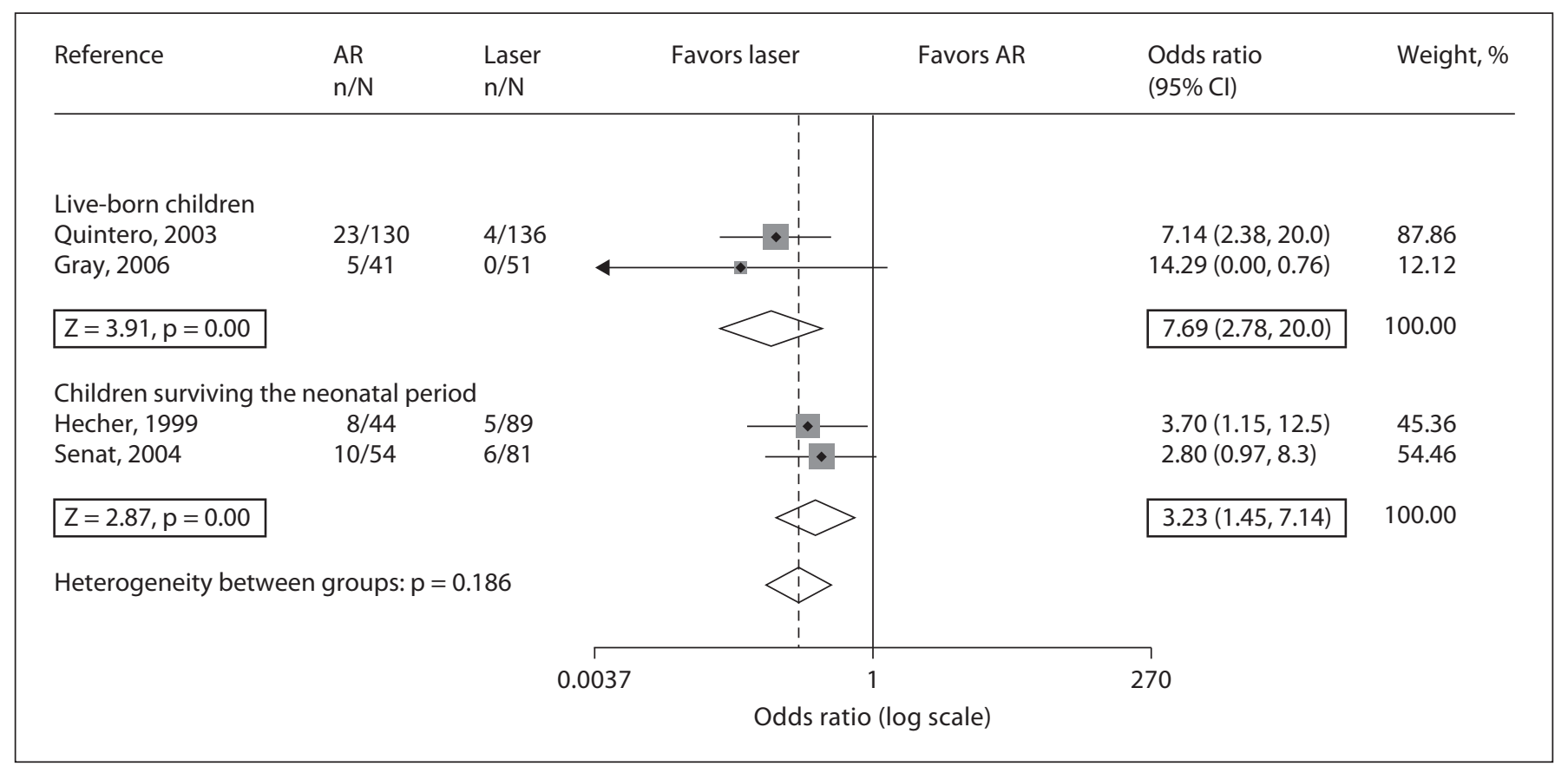

Fig. 2. Fixed effect analysis of severe cerebral injury after amnioreduction versus laser surgery.

no significant difference in $\mathrm{CPVL} \geq \mathrm{II}$ in live-born children treated with either AR or laser (OR 2.08, 95\% CI $0.86-5.00, \mathrm{p}=0.10$; fig. 3 ).

Two studies reported on the incidence of IVH $\geq$ III in live-born children $[15,20]$. Data were available from 136 children treated with AR versus 144 children treated with laser [15]. The OR demonstrated no significant difference in IVH $\geq I I I$ in live-born children treated with either AR or laser (OR 3.56, 95\% CI 0.82-14.29, p = 0.09; fig. 4). Senat and colleagues identified eight (8/95) cases of IVH $\geq$ III in live-born children in their AR group versus two live-born children (2/93) in their laser group. Of these ten cases, only one child, treated with laser, was alive at six months of age [15]. According to Gray and colleagues, none of the live-born children developed IVH $\geq$ III in their first week of life [20].

There were insufficient long-term outcome data to estimate the odds of NDI as a composite outcome. In their original article, Salomon and colleagues did not report individual observations of cognitive developmental delay as measured with the Ages and Stages Questionnaire (ASQ) and the Wechsler Intelligence Scale (WISC-IV) [16]. Salomon and colleagues did provide individual observations of CP, blindness or deafness. Data were available from 41 children treated with AR and 69 children treated with laser [16]. At 6-year follow-up, four children presented with $\mathrm{CP}$, one child was blind and one child was deaf in the AR group $(15 \% ; 6 / 41)$ versus six children with $\mathrm{CP}$, two children with blindness, and one child with deafness in the laser group $(13 \%$; 9/69). The absence of differences in long-term outcome was probably due to the significant higher neonatal death rate in the AR group, that is, $36 \%(26 / 73)$ versus $15 \%(13 / 86)$ in the laser group. Kaplan-Meier curves showed that the probability of survival without major neurological impairment was lower with $\mathrm{AR}$, adjusted for TTTS stage (hazard ratio $0.61,95 \%$ CI $0.41-0.90, p=0.01$ ) [16]. Individual results of early brain imaging of these children were not reported.

Since the number of included studies was too small for reliable assessment, construction and analysis of the funnel plot was precluded.

\section{Discussion}

The objective of our systematic review and meta-analysis was to evaluate severe cerebral injury and long-term impairment in $\mathrm{MC}$ twins treated with AR compared to laser surgery for TTTS. We found an ample seven-fold higher risk of severe cerebral injury in live-born children 


\begin{tabular}{|c|c|c|c|c|c|c|}
\hline Reference & $\begin{array}{l}\mathrm{AR} \\
\mathrm{n} / \mathrm{N}\end{array}$ & $\begin{array}{l}\text { Laser } \\
\mathrm{n} / \mathrm{N}\end{array}$ & Favors laser & Favors AR & $\begin{array}{l}\text { Odds ratio } \\
(95 \% \mathrm{Cl})\end{array}$ & Weight, \% \\
\hline \multicolumn{7}{|l|}{ Live-born children } \\
\hline Senat, 2004 & $14 / 95$ & $8 / 93$ & & & $1.85(0.73,4.55)$ & 91.52 \\
\hline Gray, 2006 & $3 / 41$ & $0 / 51 \longleftarrow$ & & & $8.33(0.40,100)$ & 8.48 \\
\hline \multirow[t]{3}{*}{$Z=1.63, p=0.10$} & & & & & $2.08(0.86,5.00)$ & 100.00 \\
\hline & & 0.00598 & & & 167 & \\
\hline & \multicolumn{6}{|c|}{ Odds ratio (log scale) } \\
\hline
\end{tabular}

Fig. 3. Fixed effect analysis of cystic periventricular leukomalacia in amnioreduction versus laser surgery.

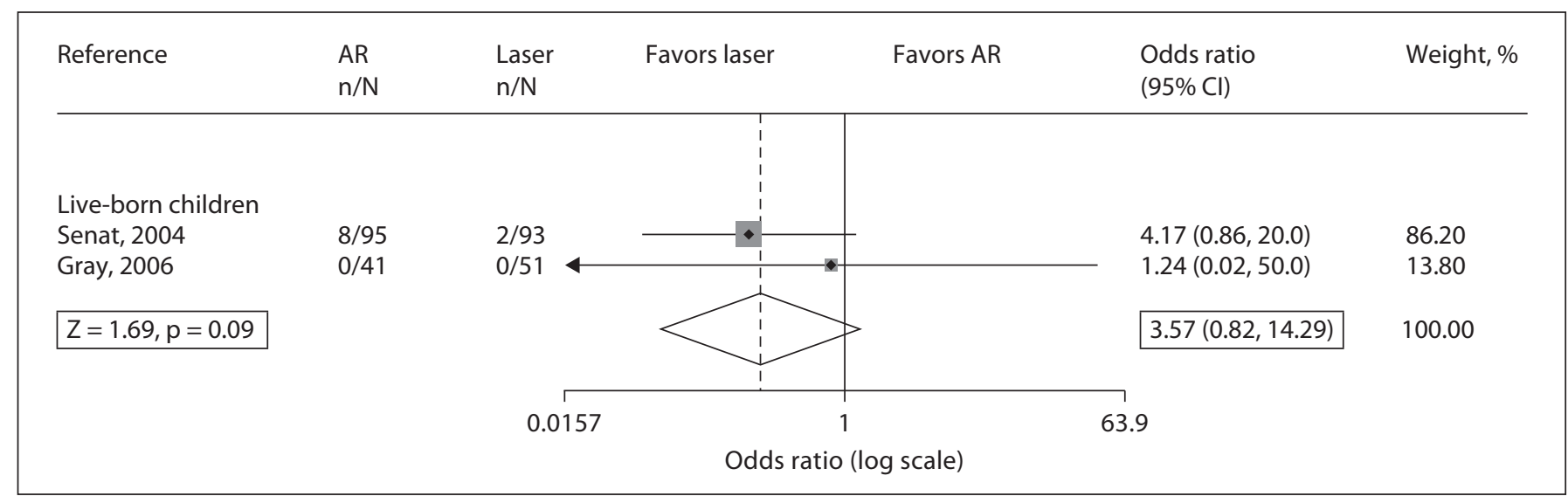

Fig. 4. Fixed effect analysis of intraventricular hemorrhage in amnioreduction versus laser surgery.

treated with AR compared to laser surgery. In children surviving the neonatal period, the odds were three times higher following AR versus laser. Detailed analysis per type of severe cerebral injury demonstrated no significant difference between treatments regarding the incidence of $\mathrm{CPVL} \geq \mathrm{II}$ and IVH $\geq$ III. Importantly, there were not enough follow-up data to analyze long-term neurodevelopmental impairment in children treated with AR compared to laser surgery.

Roberts and colleagues showed in their Cochrane review of only one trial that more children were alive without neurological abnormality at six months following laser surgery compared to AR (RR 1.66, 95\% CI 1.17-2.35 adjusted for clustering, one trial) [2]. They reported no difference in the children alive at six months with neuro- logical abnormality between interventions (RR 0.58, 95\% CI $0.18-1.86$ adjusted for clustering, one trial). The authors suggest that this might be secondary to plasticity of the developing brain or the demise of more severely affected fetuses. No data were available on outcome beyond six months at the time of writing their Cochrane review. We aimed to present the full range of the research to date and included case series as well, with a longer follow-up period.

Rossi and D'Addario showed in their meta-analysis of four studies comparing AR to laser that fetuses treated with AR were less likely to survive when compared to laser (overall survival: OR 2.04, 95\% CI 1.52-2.76, p < 0.0001 ; neonatal death: OR $0.24,95 \%$ CI $0.15-0.40$, p < 0.001 ) [13]. However, among these four studies, two stud- 
early brain imaging in order to reliably estimate the effect on later development. It is important to continuously assess child development including formal psychological testing and standardized measures of well documented psychometric quality, with increasing reliability of results with increasing age of the children. Table 3 represents a proposition for future research.

\section{Conclusion}

Setting up a new RCT with long-term follow-up after $A R$ versus laser surgery is not ethical, since higher overall survival rates and better perinatal outcomes have already been established with laser surgery. However, long-term follow-up with emphasis on child cognitive, socioemotional development and quality of life is indispensable for conducting future RCTs in all fields of fetal medicine, in order to implement new techniques.

\section{Acknowledgements}

J.M.M. van Klink was financially supported by a grant from the Willem-Alexander Kinder Fonds (Leiden, The Netherlands).

\section{Disclosure Statement}

None of the authors have a conflict of interest.

\section{References}

1 Quintero RA, Morales WJ, Allen MH, Bornick PW, Johnson PK, Kruger M: Staging of twin-twin transfusion syndrome. J Perinatol 1999; 19:550-555.

2 Roberts D, Neilson JP, Kilby M, Gates S: Interventions for the treatment of twin-twin transfusion syndrome. Cochrane Database Syst Rev 2008;CD002073.

3 Lopriore E, Oepkes D, Walther F: Neonatal morbidity in twin-twin transfusion syndrome. Early Hum Dev 2011;87:595-599.

4 Moher D, Liberati A, Tetzlaff J, Altman DG; PRISMA Group: Preferred reporting items for systematic reviews and meta-analyses: the PRISMA statement. J Clin Epidemiol 2009;62:1006-1012.

5 Wittmann BK, Baldwin VJ, Nichol B: Antenatal diagnosis of twin transfusion syndrome by ultrasound. Obstet Gynecol 1981; 58:123-127.

6 Volpe JJ: Germinal matrix-intraventricular hemorrhage of the premature infant; in Volpe JJ (ed): Neurology of the Newborn. Philadelphia, Saunders, 1995, pp 403-463.

-7 de Vries LS, Eken P, Dubowitz LM: The spectrum of leukomalacia using cranial ultrasound. Behav Brain Res 1992;49:1-6.

8 Levene MI: Measurement of the growth of the lateral ventricles in preterm infants with real-time ultrasound. Arch Dis Child 1981; 56:900-904.
9 Higgins JP, Thompson SG, Deeks JJ, Altman DG: Measuring inconsistency in meta-analyses. BMJ 2003;327:557-560.

10 DerSimonian R, Laird N: Meta-analysis in clinical trials. Control Clin Trials 1986;7: 177-188.

-11 Egger M, Davey Smith G, Schneider M, Minder C: Bias in meta-analysis detected by a simple, graphical test. BMJ 1997;315:629634.

$\$ 12$ Wagner MM, Lopriore E, Klumper FJ, Oepkes D, Vandenbussche FP, Middeldorp JM: Short- and long-term outcome in stage 1 twin-to-twin transfusion syndrome treated with laser surgery compared with conservative management. Am J Obstet Gynecol 2009;201:286.

13 Rossi AC, D’Addario V: Laser therapy and serial amnioreduction as treatment for twintwin transfusion syndrome: a metaanalysis and review of literature. Am J Obstet Gynecol 2008;198:147-152.

14 Lenclen R, Paupe A, Ciarlo G, Couderc S, Castela F, Ortqvist L, Ville Y: Neonatal outcome in preterm monochorionic twins with twin-to-twin transfusion syndrome after in trauterine treatment with amnioreduction or fetoscopic laser surgery: comparison with dichorionic twins. Am J Obstet Gynecol 2007;196:450-457.

15 Senat MV, Deprest J, Boulvain M, Paupe A, Winer N, Ville Y: Endoscopic laser surgery versus serial amnioreduction for severe twin-to-twin transfusion syndrome. N Engl J Med 2004;351:136-144.
16 Salomon LJ, Ortqvist L, Aegerter P, Bussieres L, Staracci S, Stirnemann JJ, Essaoui M, Bernard JP, Ville Y: Long-term developmental follow-up of infants who participated in a randomized clinical trial of amniocentesis vs. laser photocoagulation for the treatment of twin-to-twin transfusion syndrome. Am J Obstet Gynecol 2010;203:444-447.

17 Lenclen R, Ciarlo G, Paupe A, Bussieres L, Ville Y: Neurodevelopmental outcome at 2 years in children born preterm treated by amnioreduction or fetoscopic laser surgery for twin-to-twin transfusion syndrome: comparison with dichorionic twins. Am J Obstet Gynecol 2009;201:291-295.

- 18 Hecher K, Plath H, Bregenzer T, Hansmann M, Hackeloer BJ: Endoscopic laser surgery versus serial amniocenteses in the treatment of severe twin-twin transfusion syndrome. Am J Obstet Gynecol 1999;180:717-724.

19 Quintero RA, Dickinson JE, Morales WJ, Bornick PW, Bermudez C, Cincotta R, Chan FY, Allen MH: Stage-based treatment of twin-twin transfusion syndrome. Am J Obstet Gynecol 2003;188:1333-1340.

20 Gray PH, Cincotta R, Chan FY, Soong B: Perinatal outcomes with laser surgery for twin-twin transfusion syndrome. Twin Res Hum Genet 2006;9:438-443. 